\title{
Spin response of a normal Fermi liquid with noncentral interactions
}

\author{
C. J. Pethick 1,2 , a and A. Schwenk $k^{3, \text {, }}$ \\ ${ }^{1}$ The Niels Bohr International Academy, The Niels Bohr Institute, \\ Blegdamsvej 17, DK-2100 Copenhagen Ø, Denmark \\ ${ }^{2}$ NORDITA, Roslagstullsbacken 21, 10691 Stockholm, Sweden \\ ${ }^{3}$ TRIUMF, 4004 Wesbrook Mall, Vancouver, BC, V6T 2A3, Canada
}

\begin{abstract}
We consider the spin response of a normal Fermi liquid with noncentral interactions under conditions intermediate between the collisionless and hydrodynamic regimes. This problem is of importance for calculations of neutrino properties in dense matter. By expressing the deviation of the quasiparticle distribution function from equilibrium in terms of eigenfunctions of the transport equation under the combined influence of collisions and an external field, we derive a closed expression for the spin-density-spin-density response function and compare its predictions with that of a relaxation time approximation. Our results indicate that the relaxation time approximation is reliable for neutrino properties under astrophysically relevant conditions.

PACS numbers: 21.65.-f, 26.50.+x, 33.25.+k, 67.30.em
\end{abstract}

\section{INTRODUCTION}

Neutrino scattering, production, and annihilation rates are key physical quantities in understanding stellar collapse, neutron star formation, and supernovae, and they are determined by the spin, isospin and density responses of nuclear matter [1]. The challenge is to include the effects of collisions between nucleons and mean-field effects in a consistent way. This problem has been approached in the framework of Landau's theory of normal Fermi liquids 2] but, in the detailed calculations performed to date, the collision integral has been replaced by a simple expression with a single relaxation time [3].

In this paper we consider the spin response at long wavelengths. This problem is well understood in two limiting cases. The first is the collisionless regime, where the collision rate is small compared with the frequency $\omega$ of the field, and collisional effects may be included perturbatively 2]. When collisions cannot be taken into account perturbatively, they give rise to the LandauPomeranchuk-Migdal effect, which in the language of perturbation theory is due to energy denominators acquiring an imaginary part due to collisional damping of excitations [4].

The second regime that is well understood is the hydrodynamic one, where the collision rate is large compared with the frequency. In this case, the transport equation for quasiparticles may be solved exactly using the methods developed by Jensen, Smith and Wilkins [5] and by Brooker and Sykes [6]. In astrophysical applications, conditions intermediate between these two limiting cases arise. Related problems for the spin-independent part of the response, corresponding to distortions of the quasiparticle distribution in momentum space with an angular dependence proportional to a spherical har-

\footnotetext{
*E-mail: pethick@nbi.dk

†E-mail: schwenk@triumf.ca
}

monic, have previously been attacked using variational methods. These were developed in the context of the frequency-dependent electrical conductivity and magnetoresistance of a normal metal [7], and provide upper and lower bounds for the imaginary part of the response. These methods were subsequently exploited to obtain upper and lower bounds on the response of the quadrupolar distortion of the quasiparticle distribution, which is a key feature in calculations of the attenuation of sound in the regime intermediate between the first and zero sound [8].

Exact expressions for relaxational modes of a Fermi liquid have previously been derived in the regime intermediate between the collisionless and hydrodynamic ones in Ref. 9]. In that paper, eigenfunctions of the Fourier transform (with respect to time) of the Boltzmann transport equation for quasiparticles were found.

In the present paper we extend the work on exact solutions to include an external field, in contrast to most earlier work with exact solutions which considered transport coefficients in the hydrodynamic limit, where the effects of the external field drop out [5, 6], or involved the spectra of relaxational modes and collective modes in the absence of an external field [9]. We use the eigenfunctions obtained in Ref. [9] to derive a closed expression for the spin-density-spin-density response valid generally. We compare our results with those of a single relaxation time approximation, with a relaxation time chosen to reproduce the correct behavior in the collisionless regime, and find that for astrophysically relevant conditions, the response function given by the relaxation time approximation differs from the exact result by less than $10 \%$.

\section{BASIC FORMALISM}

We calculate the response of a normal Fermi liquid to a magnetic field in the $z$-direction that is spatially homogeneous and varies in time as $\mathrm{e}^{-\mathrm{i} \omega t}$. We assume that the quasiparticle density matrix and the quasiparticle energy may be taken to be diagonal in $\sigma_{z}$. In doing so, 
we neglect the fact that noncentral components of the interaction, which are very important in systems of nucleons, can create nonzero $x$ - and $y$-components of the spin-dependent part of the quasiparticle density matrix. In addition, we neglect tensor components of the magnetic moment. In the presence of a uniform magnetic field, these would give rise to a quadrupolar distortion of the quasiparticle distribution as a function of direction in momentum space [10]. Therefore we write the change in the energy $\delta \varepsilon_{\mathbf{p} \sigma}$ of a quasiparticle with momentum $\mathbf{p}$ and $z$-component of the spin $(\sigma= \pm 1)$ due to the application of the external field $U_{z}$ as

$$
\delta \varepsilon_{\mathbf{p} \sigma}=\sigma U_{z},
$$

and denote the quasiparticle distribution function as $n_{\mathbf{p} \sigma}$.

We start from the quasiparticle kinetic equation, which for spatially uniform conditions is given by

$$
\frac{\partial n_{\mathbf{p} \sigma}}{\partial t}=I\left[n_{\mathbf{p}^{\prime} \sigma^{\prime}}\right]
$$

where $I\left[n_{\mathbf{p}^{\prime} \sigma^{\prime}}\right]$ is the collision integral. In normal Fermi liquids at low temperatures, the dominant collisions are those between pairs of quasiparticles and, in a compact notation in which $\mathbf{p}_{i} \sigma_{i}$ is denoted simply by $i$, Eq. (2) takes the form (see Ref. [11] for details)

$$
\begin{aligned}
\frac{\partial n_{1}}{\partial t} & =-n_{1} 2 \pi \sum_{234} \frac{1}{2}|\langle 12|\mathcal{A}| 34\rangle|^{2} n_{2}\left(1-n_{3}\right)\left(1-n_{4}\right) \delta_{\varepsilon} \delta_{\mathbf{p}} \\
& +\left(1-n_{1}\right) 2 \pi \sum_{234} \frac{1}{2}|\langle 34|\mathcal{A}| 12\rangle|^{2}\left(1-n_{2}\right) n_{3} n_{4} \delta_{\varepsilon} \delta_{\mathbf{p}}
\end{aligned}
$$

where $\langle 12|\mathcal{A}| 34\rangle$ is the quasiparticle scattering amplitude, $\delta_{\varepsilon} \delta_{\mathbf{p}}$ is shorthand for $\delta\left(\varepsilon_{1}+\varepsilon_{2}-\varepsilon_{3}-\varepsilon_{4}\right) \delta\left(\mathbf{p}_{1}+\mathbf{p}_{2}-\mathbf{p}_{3}-\mathbf{p}_{4}\right)$ and $\sum_{i}=\sum_{\sigma_{i}} \int d \mathbf{p}_{i} /(2 \pi)^{3}$. The two quasiparticles in the scattering process are of the same species and the factor $1 / 2$ avoids double counting of final states when momenta are freely summed over. In addition, we assume that $\omega \ll T$, so we do not include $\omega$ in the energyconserving delta function [12].

The quasiparticle energies include contributions due to the external field and due to "molecular fields" resulting from interactions with other quasiparticles,

$$
\varepsilon_{\mathbf{p} \sigma}=\varepsilon_{\mathbf{p} \sigma}^{0}+\delta \varepsilon_{\mathbf{p} \sigma}
$$

where $\varepsilon_{\mathbf{p} \sigma}^{0}$ denotes the quasiparticle energy in equilibrium in the absence of the magnetic field and $\delta \varepsilon_{\mathbf{p} \sigma}$ includes the interaction with the magnetic field, Eq. (1), and the contribution due to quasiparticle interactions $f_{12}$,

$$
\delta \varepsilon_{1}=\sigma_{1} U_{z}+\sum_{2} f_{12} \delta n_{2}
$$

Tensor components of the Landau quasiparticle interaction are generally small in nuclear matter and we therefore neglect them. In this case the response of the quasiparticle distribution to a spatially uniform magnetic field is isotropic and the interaction energy reduces to $g_{0} S$, where $S=\sum_{1} \sigma_{1} \delta n_{1}$ is the total spin and $g_{0}$ (or $f_{0}^{a}$ in the quantum liquids' literature [13]) is the isotropic component of the spin-dependent interaction between quasiparticles. The change in the quasiparticle energy is thus

$$
\delta \varepsilon_{1}=\sigma_{1}\left(U_{z}+g_{0} S\right)
$$

We now linearize Eq. (3) about the equilibrium distribution function

$$
n_{i}^{0}=\frac{1}{\mathrm{e}^{\left(\varepsilon_{i}^{0}-\mu\right) / T}+1}
$$

in the standard way [11, Sect. 1.2.4] taking into account the variation of the quasiparticle energies in the delta function. Here $\mu$ is the chemical potential in equilibrium. It is convenient to introduce the quantity

$$
\delta \bar{n}_{i}=n_{i}-n^{0}\left(\varepsilon_{i}\right)=n_{i}-n_{i}^{0}-\frac{\partial n_{i}^{0}}{\partial \varepsilon_{i}^{0}} \delta \varepsilon_{i},
$$

which represents the difference between the quasiparticle distribution function and the Fermi function evaluated for the actual quasiparticle energy, not the one in equilibrium. Linearizing Eq. (3) leads to

$$
\begin{aligned}
\frac{\partial \delta \bar{n}_{1}}{\partial t}+\sigma_{1} \frac{\partial n_{1}^{0}}{\partial \varepsilon_{1}^{0}} \frac{\partial\left(U_{z}+g_{0} S\right)}{\partial t} & =-\frac{2 \pi}{T} \sum_{234} \frac{1}{2}|\langle 12|\mathcal{A}| 34\rangle|^{2} \delta_{\mathbf{p}} \delta_{x}\left(\prod_{i=1}^{4} \frac{1}{2 \cosh \left(x_{i} / 2\right)}\right) \\
& \times\left[2 \cosh \left(x_{1} / 2\right) \Xi_{1}+2 \cosh \left(x_{2} / 2\right) \Xi_{2}-2 \cosh \left(x_{3} / 2\right) \Xi_{3}-2 \cosh \left(x_{4} / 2\right) \Xi_{4}\right],
\end{aligned}
$$

where $x_{i}=\left(\varepsilon_{i}^{0}-\mu\right) / T$, so that one has $\left(\partial n_{i}^{0} / \partial \varepsilon_{i}^{0}\right)^{-1}=$ $-T\left[2 \cosh \left(x_{i} / 2\right)\right]^{2}$, and $\delta_{x}=\delta\left(x_{1}+x_{2}-x_{3}-x_{4}\right)$. In addition, we have introduced $\Xi_{i}=2 \cosh \left(x_{i} / 2\right) \delta \bar{n}_{i}$, which simplifies the subsequent calculations. In writing the right-hand side of Eq. (9) in this form, we have used the property that for interactions invariant under time reversal $\left\langle\mathbf{p}_{1} \sigma_{1}, \mathbf{p}_{2} \sigma_{2}|\mathcal{A}| \mathbf{p}_{3} \sigma_{3}, \mathbf{p}_{4} \sigma_{4}\right\rangle=\left(\left\langle-\mathbf{p}_{3}-\sigma_{3},-\mathbf{p}_{4}-\right.\right.$ $\left.\left.\sigma_{4}|\mathcal{A}|-\mathbf{p}_{1}-\sigma_{1},-\mathbf{p}_{2}-\sigma_{2}\right\rangle\right)^{*}$, and therefore, since the dis- 
tribution function is isotropic in momentum space, one may combine the two terms in Eq. (3). After Fourier transforming with respect to time, Eq. (9) may be recast as an inhomogeneous integral equation for $\Xi$,

$$
\begin{aligned}
\frac{\sigma_{1}\left(U_{z}+g_{0} S\right)}{2 T \cosh \left(x_{1} / 2\right)} & =\Xi_{1}+\frac{\mathrm{i}}{\omega} \frac{2 \pi}{T} \sum_{234} \frac{1}{2}|\langle 12|\mathcal{A}| 34\rangle|^{2} \delta_{\mathbf{p}} \delta_{x}\left(\prod_{i=2}^{4} \frac{1}{2 \cosh \left(x_{i} / 2\right)}\right) \\
& \times\left[2 \cosh \left(x_{1} / 2\right) \Xi_{1}+2 \cosh \left(x_{2} / 2\right) \Xi_{2}-2 \cosh \left(x_{3} / 2\right) \Xi_{3}-2 \cosh \left(x_{4} / 2\right) \Xi_{4}\right] .
\end{aligned}
$$

For $T \ll \varepsilon_{\mathrm{F}}$, scattering processes are strongest for quasiparticle states in the vicinity of the Fermi surface. As a consequence, integrals over the magnitudes of the momenta may be decoupled from integrals over quasiparticle energies, and the scattering amplitudes involved can be replaced by their values for all quasiparticle momenta on the Fermi surface. In the literature on quantum liquids it is customary to write [11, 14]

$$
d \mathbf{p}_{2}=p_{2}^{2} d p_{2} d \cos \theta d \phi_{2}
$$

where $\theta$ is the angle between $\mathbf{p}_{1}$ and $\mathbf{p}_{2}$ and $\phi_{2}$ is an azimuthal angle, and

$$
d \mathbf{p}_{3}=p_{3}^{2} d p_{3} d \cos \theta_{3} d \phi
$$

where $\theta_{3}$ is the angle between $\mathbf{p}_{3}$ and the total momentum $\mathbf{P}=\mathbf{p}_{1}+\mathbf{p}_{2}$ of the incoming quasiparticles and $\phi$ is the angle between the plane containing $\mathbf{p}_{1}$ and $\mathbf{p}_{2}$ and that containing $\mathbf{p}_{3}$ and $\mathbf{p}_{4}$. The integral over $\theta_{3}$ can be converted into one over $\varepsilon_{4}$ by using the fact that $\mathbf{p}_{4}=\mathbf{P}-\mathbf{p}_{3}$, and therefore

$$
p_{4}^{2}=P^{2}+p_{3}^{2}-2 P p_{3} \cos \theta_{3},
$$

from which is follows that

$$
p_{4} d p_{4}=-P p_{3} d \cos \theta_{3},
$$

when $p_{3}$ is held fixed, or

$$
\left|\frac{\partial \cos \theta_{3}}{\partial \varepsilon_{4}}\right|=\frac{m^{*}}{P k_{\mathrm{F}}} \quad \text { for fixed } p_{3}
$$

Thus we may write

$$
d \mathbf{p}_{2} d \mathbf{p}_{3}=\frac{m^{* 3} k_{\mathrm{F}}}{P} d \varepsilon_{2} d \varepsilon_{3} d \varepsilon_{4} d \cos \theta d \phi d \phi_{2}
$$

where $P=2 k_{\mathrm{F}} \cos (\theta / 2)$.

Next we express $\Xi$ as

$$
\Xi_{i}=\sigma_{i} \frac{\left(U_{z}+g_{0} S\right)}{T} \xi\left(x_{i}\right)
$$

substitute this expression into Eq. (10), multiply the result by $\sigma_{1}$, average over $\sigma_{1}$, and perform the integrals. The integral over $\phi_{2}$ gives $2 \pi$ and the term involving $\Xi_{1}$ is proportional to the integral $K_{3}$, Eq. (A5). One thus obtains the linearized quasiparticle transport equation,

$$
\frac{1}{2 \cosh \left(x_{1} / 2\right)}=\frac{\mathrm{i}}{2 \omega \tau_{0}}\left(x_{1}^{2}+\pi^{2} \zeta^{2}\right) \xi\left(x_{1}\right)-\frac{\mathrm{i} \lambda_{\sigma}}{\omega \tau_{0}} \int_{-\infty}^{\infty} \frac{d x_{2} d x_{3} d x_{4} \delta_{x}}{4 \cosh \left(x_{2} / 2\right) \cosh \left(x_{4} / 2\right)} \xi\left(x_{3}\right) .
$$

Here we have introduced the quantities

$$
\begin{aligned}
\zeta^{2} & =1-\mathrm{i} \frac{2 \omega \tau_{0}}{\pi^{2}}, \\
\frac{1}{\tau_{0}} & =\frac{\pi}{16} \frac{T^{2}}{k_{\mathrm{F}} v_{\mathrm{F}}}\left\langle\operatorname{Tr}_{\sigma}|\langle 12|\mathcal{A}| 34\rangle|^{2}\right\rangle, \\
\lambda_{\sigma} & =\frac{\left\langle\operatorname{Tr}_{\sigma}|\langle 12|\mathcal{A}| 34\rangle|^{2} \sigma_{1}\left(-\sigma_{2}+\sigma_{3}+\sigma_{4}\right)\right\rangle}{\left\langle\operatorname{Tr}_{\sigma}|\langle 12|\mathcal{A}| 34\rangle|^{2}\right\rangle},
\end{aligned}
$$

where the trace $\operatorname{Tr}_{\sigma}$ denotes a sum over all spins $\sigma_{i}$ and the average is defined by

$$
\langle\ldots\rangle=\frac{1}{8 \pi} \int_{0}^{\pi} \frac{\sin \theta d \theta}{\cos (\theta / 2)} \int_{0}^{2 \pi} d \phi \ldots
$$

and $v_{\mathrm{F}}=k_{\mathrm{F}} / m^{*}$ is the Fermi velocity. The factor of $1 /(8 \pi)$ ensures that the average is normalized to unity [15]. The first term on the right-hand side of Eq. (18) includes the contribution from the integral over $x_{2}, x_{3}$ and $x_{4}$ given by the function $K_{3}$, Eq. (A5), in Appendix $\mathrm{A}$. The integrals over $x_{3}$ and $x_{4}$ in Eq. (18) yield the standard form for the kernel, given by the function $K_{2}$, Eq. (A4). However, for the Fourier transformation 
in the next section, it is simpler if the integral is left in the original form.

Physically, the quantity $2 \tau_{0} / \pi^{2}$ is the relaxation time of a quasiparticle in a particular momentum state at the Fermi surface, if deviations of the quasiparticle distribution from equilibrium in all other states vanish. For an interaction with only central terms, the expression, Eq. (20), reduces to that derived in the context of liquid ${ }^{3} \mathrm{He}$ [16]. The quantity $1-\lambda_{\sigma}$ is a measure of the effectiveness of collisions in relaxing the spin of the quasiparticle: if spin is conserved in collisions, $\sigma_{1}+\sigma_{2}=\sigma_{3}+\sigma_{4}$ and therefore $\lambda_{\sigma}=1$, while if $\lambda_{\sigma}=0$, the integral term in Eq. (18) vanishes and the equation becomes an algebraic one, whose physical content is equivalent to a relaxation time approximation with a relaxation time that depends on the quasiparticle energy, as we will see in Sect. IV.

In applications it is sometimes convenient to express the scattering amplitudes in terms of the momentum transfers $\mathbf{k}=\mathbf{p}_{1}-\mathbf{p}_{3}$ and $\mathbf{k}^{\prime}=\mathbf{p}_{1}-\mathbf{p}_{4}$. For quasiparticles at the Fermi surface these satisfy the condition

$$
P^{2}+k^{2}+k^{\prime 2}=4 k_{\mathrm{F}}^{2}
$$

The magnitudes of the momentum transfers are given by $k=2 k_{\mathrm{F}} \sin (\theta / 2) \sin (\phi / 2)$ and $k^{\prime}=2 k_{\mathrm{F}} \sin (\theta / 2) \cos (\phi / 2)$ and the Jacobian for the transformation is

$$
\left|\frac{\partial(\cos \theta, \phi)}{\partial\left(k, k^{\prime}\right)}\right|=\frac{2}{k_{\mathrm{F}}^{2}}
$$

and therefore [3]

$$
\langle\ldots\rangle=\frac{1}{\pi} \int_{0}^{2 k_{\mathrm{F}}} \frac{d k}{k_{\mathrm{F}}} \int_{0}^{2 k_{\mathrm{F}}} \frac{d k^{\prime}}{k_{\mathrm{F}}} \frac{k_{\mathrm{F}} \Theta\left(4 k_{\mathrm{F}}^{2}-k^{2}-k^{2}\right)}{\sqrt{4 k_{\mathrm{F}}^{2}-k^{2}-k^{\prime 2}}} \ldots
$$

When effects of the medium on scattering amplitudes are taken into account, the scattering amplitude will also depend on the total momentum of the incoming quasiparticles, and the natural variables for scattering of quasiparticles at the Fermi surface are $P$ and $\phi$. In terms of these the average, Eq. (22), takes on the simple form

$$
\langle\ldots\rangle=\int_{0}^{2 k_{\mathrm{F}}} \frac{d P}{2 k_{\mathrm{F}}} \int_{0}^{2 \pi} \frac{d \phi}{2 \pi} \ldots
$$

In the next section, we solve Eq. (18) for $\xi$, from which one can calculate the total spin $S$,

$$
\begin{aligned}
S & =\sum_{1} \sigma_{1} \delta n_{1}=\sum_{1} \sigma_{1}\left(\delta \bar{n}_{1}+\sigma_{1} \frac{\partial n_{1}^{0}}{\partial \varepsilon_{1}^{0}}\left(U_{z}+g_{0} S\right)\right) \\
& =\sum_{1} \sigma_{1}\left(\frac{\Xi_{1}}{2 \cosh \left(x_{1} / 2\right)}+\sigma_{1} \frac{\partial n_{1}^{0}}{\partial \varepsilon_{1}^{0}}\left(U_{z}+g_{0} S\right)\right) .
\end{aligned}
$$

Since $\Xi$ is proportional to $U_{z}+g_{0} S$, this equation shows that $S$ can be written in the form

$$
S=-\chi_{\sigma} U_{z}
$$

where the spin-density-spin-density response function $\chi_{\sigma}$ is given by

$$
\chi_{\sigma}=\frac{X_{\sigma}}{1+g_{0} X_{\sigma}},
$$

and $X_{\sigma}=-S /\left(U_{z}+g_{0} S\right)$ denotes the response function in the absence of mean-field effects $\left(g_{0}=0\right)$. Using Eq. (17), it follows from Eq. (27) that

$$
X_{\sigma}=N(0)\left(1-\int_{-\infty}^{\infty} \frac{d x}{2 \cosh (x / 2)} \xi(x)\right)
$$

with $N(0)=-\sum_{1} \partial n_{1}^{0} / \partial \varepsilon_{1}^{0}$ being the density of states at the Fermi surface.

\section{SOLUTION OF TRANSPORT EQUATION}

To solve the linearized transport equation, Eq. (18), we follow the methods developed by Brooker and Sykes [9] and express the solution $\xi$ in the form

$$
\xi(x)=\sum_{r=0}^{\infty} a_{r} \Phi_{r}(x)
$$

where $\Phi_{r}(x)$ are eigenfunctions of the homogeneous equation,

$$
\begin{aligned}
\left(x_{1}^{2}+\right. & \left.\pi^{2} \zeta^{2}\right) \Phi_{r}\left(x_{1}\right) \\
& =\frac{\Lambda_{r}}{2} \int_{-\infty}^{\infty} \frac{d x_{2} d x_{3} d x_{4} \delta_{x}}{\cosh \left(x_{2} / 2\right) \cosh \left(x_{4} / 2\right)} \Phi_{r}\left(x_{3}\right) .
\end{aligned}
$$

The eigenfunctions may be determined by Fourier transforming. We write

$$
\widetilde{\Phi}_{r}(k)=\int_{-\infty}^{\infty} d x \Phi_{r}(x) \mathrm{e}^{-\mathrm{i} k x} .
$$

By using the representation, Eq. (A3), for the delta function, one finds that the Fourier transform of the integral term reduces to the product of $\widetilde{\Phi}_{r}(k)$ and the square of the Fourier transform of $1 / \cosh (x / 2)$, given by $2 \pi / \cosh (\pi k)$. Thus the eigenfunctions satisfy

$$
-\frac{d^{2} \widetilde{\Phi}_{r}(k)}{d k^{2}}+\pi^{2} \zeta^{2} \widetilde{\Phi}_{r}(k)-\frac{2 \pi^{2} \Lambda_{r}}{\cosh ^{2}(\pi k)} \widetilde{\Phi}_{r}(k)=0,
$$

which has the same form as the Schrödinger equation for a particle moving in a one-dimensional potential $\sim$ $\operatorname{sech}^{2}(\pi k)$ with a (generally complex) energy $\sim \zeta^{2}[17]$. This is analogous to the Sturmian method in quantummechanical problems, in which one uses a basis of states with the same energy, but with different strengths of the potential [18].

In this paper we are concerned with disturbances that are even in $x$, and therefore the relevant eigenvalues are

$$
\Lambda_{r}=\frac{1}{2}(2 r+\zeta)(2 r+1+\zeta),
$$


where $r$ is positive integer or zero, and the corresponding solutions [9, p. 85] are given by

$$
\begin{aligned}
\widetilde{\Phi}_{r}(k)= & 2^{\zeta} \Gamma(1+\zeta) P_{2 r+\zeta}^{-\zeta}(\tanh (\pi k)), \\
= & \frac{(2 r) ! \Gamma(2 \zeta+1)}{\Gamma(2 r+2 \zeta+1)}[\cosh (\pi k)]^{-\zeta} C_{2 r}^{\zeta+1 / 2}(\tanh (\pi k)), \\
= & { }_{2} F_{1}(-2 r, 2 r+1+2 \zeta, 1+\zeta,[1-\tanh (\pi k)] / 2) \\
& \times[\cosh (\pi k)]^{-\zeta}
\end{aligned}
$$

where $C_{n}^{m}$ are Gegenbauer polynomials and ${ }_{2} F_{1}$ is the hypergeometric function. The corresponding eigenfunctions as a function of energy, $\Phi_{r}(x)$, may be determined by Fourier transformation, and some of their properties are described in Appendix A.

On substituting Eq. (31) into Eq. (18), multiplying by $\Phi_{r}\left(x_{1}\right)$ and integrating over $x_{1}$ one finds

$$
a_{r}=-\mathrm{i} 2 \omega \tau_{0} \frac{T_{r}}{U_{r}\left(\Lambda_{r}-\lambda_{\sigma}\right)}
$$

where, following the notation of Ref. [9], we have defined

$$
T_{r}=\int_{-\infty}^{\infty} \frac{d k \widetilde{\Phi}_{r}(k)}{\cosh (\pi k)}=\frac{\Gamma(\zeta+1) \Gamma\left(\frac{1}{2}+r+\frac{1}{2} \zeta\right) \Gamma\left(\frac{1}{2}+r\right)}{\pi \Gamma\left(1+r+\frac{1}{2} \zeta\right) \Gamma(1+r+\zeta)}
$$

and the normalizing integral is given by

$$
U_{r}=2 \pi \int_{-\infty}^{\infty} \frac{d k\left[\widetilde{\Phi}_{r}(k)\right]^{2}}{\cosh ^{2}(\pi k)}=\frac{2^{2 \zeta+1}(2 r) ![\Gamma(\zeta+1)]^{2}}{\left(2 r+\zeta+\frac{1}{2}\right) \Gamma(2 r+2 \zeta+1)}
$$

The integral in Eq. (30) therefore leads to

$$
\begin{aligned}
\int_{-\infty}^{\infty} \frac{d x \xi(x)}{2 \cosh (x / 2)} & =\sum_{r=0}^{\infty} \frac{a_{r} T_{r}}{2} \\
& =-i \omega \tau_{0} \sum_{r=0}^{\infty} \frac{\left(T_{r}\right)^{2}}{U_{r}} \frac{1}{\Lambda_{r}-\lambda_{\sigma}}
\end{aligned}
$$

and the response function $X_{\sigma}$ in the absence of mean-field effects is given by

$$
\begin{aligned}
\frac{X_{\sigma}}{N(0)} & =1+\mathrm{i} \frac{\omega \tau_{0}}{\pi^{2}} \sum_{r=0}^{\infty} \frac{\left(2 r+\zeta+\frac{1}{2}\right) \Gamma(2 r+2 \zeta+1)}{2^{2 \zeta+1}(2 r) !\left(\Lambda_{r}-\lambda_{\sigma}\right)}\left(\frac{\Gamma\left(\frac{1}{2}+r+\frac{1}{2} \zeta\right) \Gamma\left(\frac{1}{2}+r\right)}{\Gamma\left(1+r+\frac{1}{2} \zeta\right) \Gamma(1+r+\zeta)}\right)^{2} \\
& =1+\mathrm{i} \frac{2 \omega \tau_{\sigma}\left(1-\lambda_{\sigma}\right)}{3} \sum_{r=0}^{\infty} \frac{\left(2 r+\zeta+\frac{1}{2}\right) \Gamma(2 r+2 \zeta+1)}{2^{2 \zeta+1}(2 r) !\left(\Lambda_{r}-\lambda_{\sigma}\right)}\left(\frac{\Gamma\left(\frac{1}{2}+r+\frac{1}{2} \zeta\right) \Gamma\left(\frac{1}{2}+r\right)}{\Gamma\left(1+r+\frac{1}{2} \zeta\right) \Gamma(1+r+\zeta)}\right)^{2}
\end{aligned}
$$

The sum in Eq. (43) may be expressed in terms of the generalized hypergeometric function ${ }_{5} F_{4}$ but in applications it offers no advantages compared with a direct evaluation of the sum. Moreover, we write the result in terms of the physically relevant timescale in spin relaxation, $\tau_{\sigma}$, which for the case of interest here, $\omega \ll T$, is given by

$$
\tau_{\sigma}=\frac{3 \tau_{0}}{2 \pi^{2}\left(1-\lambda_{\sigma}\right)}
$$

In Ref. 3] a single relaxation time approximation was made for the collision term, and this led to the response function (in the absence of mean-field effects)

$$
X_{\sigma}^{\text {relax }}=\frac{N(0)}{1-\mathrm{i} \omega \tau_{\sigma}} .
$$

In terms of $\tau_{\sigma}$, the quantity $\zeta$ is given by

$$
\zeta^{2}=1-\mathrm{i} \frac{4 \omega \tau_{\sigma}}{3}\left(1-\lambda_{\sigma}\right)
$$

and this ensures that the relaxation time approximation, Eq. (45), reproduces the leading term i $N(0) /\left(\omega \tau_{\sigma}\right)$ in the response function for $\omega \tau_{\sigma} \gg 1$. This can be shown by observing that, in this limit, the leading contribution to the deviation function is given by $\xi^{(0)}(x)=1 /[2 \cosh (x / 2)]$. When $\omega \tau_{0}$ is large, the leading contributions to $\xi^{(1)}$ due to collisions can be calculated in perturbation theory, treating $1 /\left(\omega \tau_{0}\right)$ as small parameter, and one finds

$$
\begin{aligned}
\xi^{(1)}\left(x_{1}\right)= & -\frac{\mathrm{i}}{2 \omega \tau_{0}}\left(\left(x_{1}^{2}+\pi^{2}\right) \xi^{(0)}\left(x_{1}\right)\right. \\
& \left.-\lambda_{\sigma} \int_{-\infty}^{\infty} \frac{d x_{2} d x_{3} d x_{4} \delta_{x}}{4 \cosh \left(x_{2} / 2\right) \cosh \left(x_{4} / 2\right)} \xi^{(0)}\left(x_{3}\right)\right) \\
= & -\frac{\mathrm{i}}{2 \omega \tau_{0}}\left(x_{1}^{2}+\pi^{2}\right)\left(1-\lambda_{\sigma}\right) \xi^{(0)}\left(x_{1}\right)
\end{aligned}
$$

where we have used the fact that $1 /[2 \cosh (x / 2)]$ is the eigenfunction of the integral operator with eigenvalue 1 , see Eq. A10. From this, one finds that leading contribution to the response function is given by $X_{\sigma}=$ iN $N(0) /\left(\omega \tau_{\sigma}\right)$ at high frequency. The analogous result for spin-independent distortions of the Fermi surface was derived in Ref. [19].

\section{EXAMPLES}

One case that can be solved simply is that for $\lambda_{\sigma}=0$, and Eq. (18) then gives

$$
\xi(x)=-\frac{\mathrm{i} \omega \tau_{0}}{\left(x^{2}+\pi^{2} \zeta^{2}\right) \cosh (x / 2)} .
$$



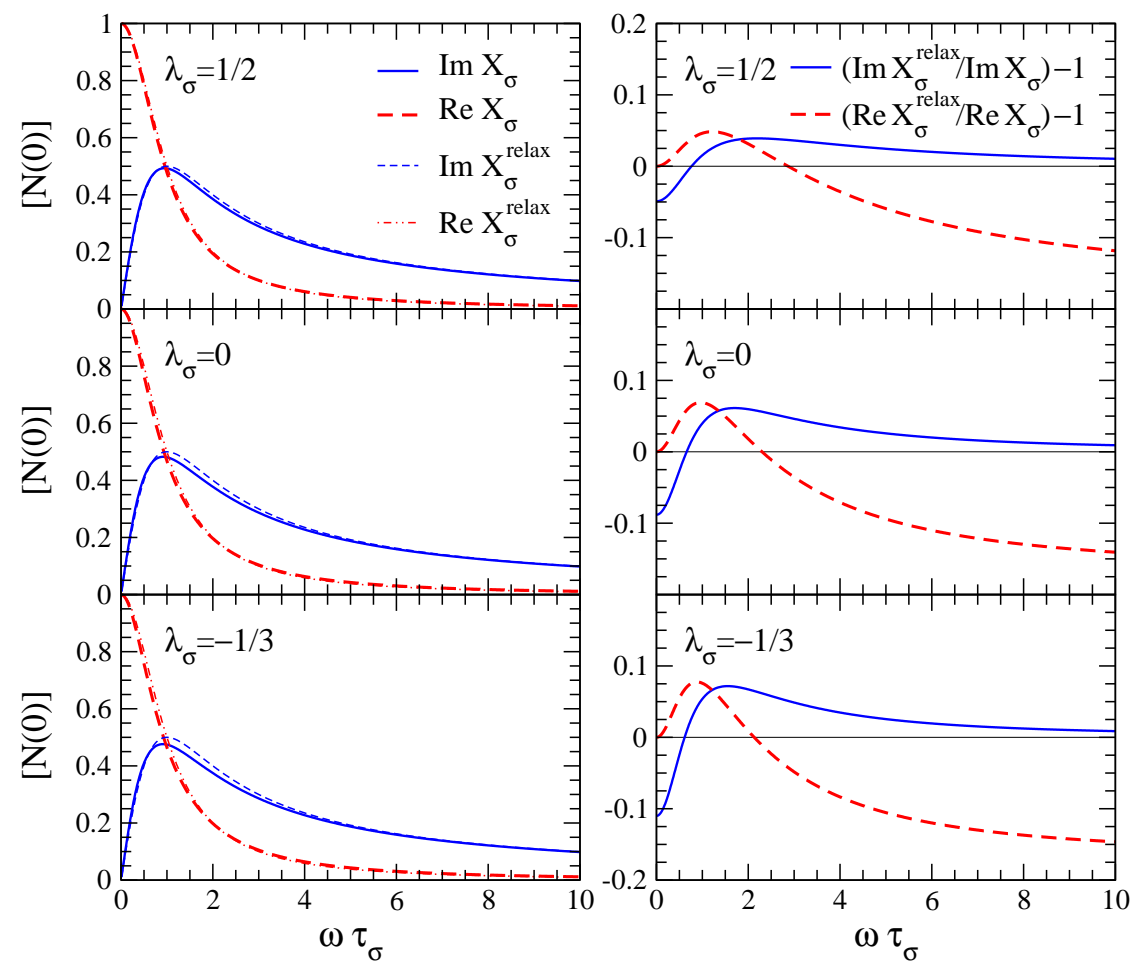

FIG. 1: (Color online) Left panels: Real and imaginary parts of the response function $X_{\sigma}$ in units of the density of states at the Fermi surface $N(0)$, for $\lambda_{\sigma}=1 / 2,0$ and $-1 / 3$, as a function of $\omega \tau_{\sigma}$. The spin relaxation time $\tau_{\sigma}$ is given by Eq. (44). For comparison, we show the response function based on the relaxation time approximation $X_{\sigma}^{\text {relax }}$. Right panels: Relative deviations of the real and imaginary parts of the relaxation time approximation and the exact $\operatorname{result},\left(\operatorname{Re} X_{\sigma}^{\mathrm{relax}} / \operatorname{Re} X_{\sigma}\right)-1$ and $\left(\operatorname{Im} X_{\sigma}^{\text {relax }} / \operatorname{Im} X_{\sigma}\right)-1$, for the same values of $\lambda_{\sigma}$.

Substituting this expression into Eq. (30) leads to the response function [20]

$$
X_{\sigma}=N(0)\left(1+\frac{\mathrm{i} \omega \tau_{0}}{\pi^{2} \zeta} \psi^{\prime}((1+\zeta) / 2)\right)
$$

where $\psi^{\prime}(z)$ is the trigamma function [21]. In this limit, numerical results using Eq. (49) agree very well with those obtained from the general expression, Eq. (43), thereby providing a check on the latter.

In the middle-left panel of Fig. 1, we show the real and imaginary parts of the response function $X_{\sigma}$ for $\lambda_{\sigma}=0$ as a function of $\omega \tau_{\sigma}$. For comparison, results based on the relaxation time approximation, Eq. (45), are also given. The physically relevant time scale that determines the transition between collisionless and hydrodynamic behavior is $\tau_{\sigma}$ which, according to Eq. (44), is shorter than $\tau_{0}$ by a factor $3 /\left(2 \pi^{2}\right) \approx 0.15$ for $\lambda_{\sigma}=0$. In the right panel of Fig. 1, the relative differences between the relaxation time approximation and the exact result are shown more clearly, by plotting $\left(\operatorname{Re} X_{\sigma}^{\text {relax }} / \operatorname{Re} X_{\sigma}\right)-1$ and $\left(\operatorname{Im} X_{\sigma}^{\mathrm{relax}} / \operatorname{Im} X_{\sigma}\right)-1$. In the top and bottom panels of Fig. 1. we present results for two other values of $\lambda_{\sigma}$, relevant for neutrino interactions and the spin response of neutron matter. These are $\lambda_{\sigma}=1 / 2$, which corresponds to a ratio of the quasiparticle relaxation time to the spin relaxation time, $\tau / \tau_{\sigma}=3 \tau_{0} /\left(2 \pi^{2} \tau_{\sigma}\right)=1 / 2$, typical for low-momentum two-nucleon interactions (see Fig. 3 in Ref. [3]) with very similar rates obtained in chiral effective field theory [22], and $\lambda_{\sigma}=-1 / 3$, which corresponds to $\tau / \tau_{\sigma}=4 / 3$, the value for the one-pion exchange approximation to nuclear interactions [3].

At low frequencies the relaxation time approximation always underestimates $\operatorname{Im} X_{\sigma}$. This may be understood in terms of the standard variational calculation of the relaxation time in the hydrodynamic limit. Generally, one can write the response function at low frequencies as

$$
X_{\sigma} \approx N(0)\left(1+\mathrm{i} \omega \tau_{\sigma}^{\text {hydro }}\right),
$$

where $\tau_{\sigma}^{\text {hydro }}$ is a characteristic relaxation time in this limit. According to Eqs. (43) and (50), the ratio of the spin relaxation times in the hydrodynamic and collisionless limits is given by

$$
\begin{aligned}
\frac{\tau_{\sigma}^{\text {hydro }}}{\tau_{\sigma}} & =\frac{4\left(1-\lambda_{\sigma}\right)}{3} \\
& \times \sum_{r=0}^{\infty} \frac{4 r+3}{(2 r+1)(2 r+2)\left[(2 r+1)(2 r+2)-2 \lambda_{\sigma}\right]} .
\end{aligned}
$$

This result may also be obtained directly by generalizing the calculations of Refs. [5, 6] to the problem of spin 


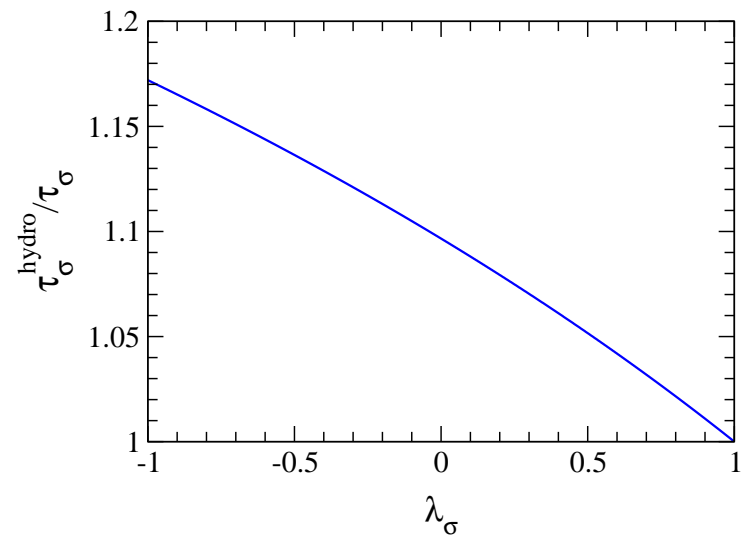

FIG. 2: Ratio of spin relaxation times in the hydrodynamic and collisionless limits $\tau_{\sigma}^{\text {hydro }} / \tau_{\sigma}$ as a function of $\lambda_{\sigma}$.

relaxation in a system with noncentral interactions. The quantity $\tau_{\sigma}$ is the simplest variational estimate of the relaxation time and consequently always underestimates the relaxation time $\tau_{\sigma}^{\text {hydro }}$ in this limit. In Fig. 2, we show the ratio $\tau_{\sigma}^{\text {hydro }} / \tau_{\sigma}$ as a function of $\lambda_{\sigma}$. For systems, in which collisions only occur between particles with the same spin projection and always change the sign of the spin projection, one has $\lambda_{\sigma}=-1$ and the lack of spin conservation is maximal. In this case, $\tau_{\sigma}^{\text {hydro }} / \tau_{\sigma} \approx 1.17$, while for $\lambda_{\sigma}=0$ the ratio is $\tau_{\sigma}^{\text {hydro }} / \tau_{\sigma}=\pi^{2} / 9 \approx 1.10$ and for $\lambda_{\sigma} \rightarrow 1$ one finds $\tau_{\sigma}^{\text {hydro }} / \tau_{\sigma} \rightarrow 1$.

The relaxation time approximation overestimates $\operatorname{Im} X_{\sigma}$ at higher frequencies. That the differences of the imaginary parts cannot have the same sign for low and high frequencies is a necessary consequence of the fact that the response functions satisfy a Kramers-Kronig relation, and therefore

$$
\begin{aligned}
\frac{2}{\pi} \int_{0}^{\infty} \frac{d \omega}{\omega}\left[\operatorname{Im} X_{\sigma}(\omega)-\operatorname{Im} X_{\sigma}^{\mathrm{relax}}(\omega)\right] \\
=\operatorname{Re} X_{\sigma}(0)-\operatorname{Re} X_{\sigma}^{\mathrm{relax}}(0)=0 .
\end{aligned}
$$

In addition, at high frequencies $\operatorname{Re} X_{\sigma}$ is always greater than the relaxation time approximation. This is due to the fact that the leading term at high frequencies is proportional to the average of the square of the collision operator, while in the relaxation time approximation this is replaced by the square of the average. For $\lambda_{\sigma}=0$, this ratio can be calculated simply, and one finds

$$
\frac{\operatorname{Re} X_{\sigma}^{\text {relax }}}{\operatorname{Re} X_{\sigma}} \rightarrow \frac{\left(\int_{-\infty}^{\infty} d x\left(x^{2}+\pi^{2}\right) \operatorname{sech}^{2}(x / 2)\right)^{2}}{\int_{-\infty}^{\infty} d x\left(x^{2}+\pi^{2}\right)^{2} \operatorname{sech}^{2}(x / 2)}=\frac{5}{6},
$$

in the limit $\omega \tau_{\sigma} \gg 1$.

In summary, our results show that the differences between the real and imaginary parts of the spin response function calculated in the relaxation time approximation and the exact result are generally less than $10 \%$ for the conditions relevant to neutrino interactions in stellar collapse, supernovae and neutron stars. We do not expect this conclusion to change when mean-field effects are included, since for the densities of interest, the Landau parameter $G_{0} \approx 0.8$ is positive and not large (see, for example, Ref. [23]). Consequently, the errors of using the relaxation time approximation are small compared to the theoretical uncertainties in determining the spin relaxation times in dense matter from nuclear forces.

\section{DISCUSSION}

We first consider applications of the spin response function to calculations of neutrino-pair bremsstrahlung and absorption and to neutrino scattering. As shown in Ref. [24], if one neglects the momentum transfer to the nuclear medium and Pauli blocking effects for neutrinos, the rates of neutrino processes when averaged over a Boltzmann distribution for the neutrinos are proportional to integrals of the form

$$
I_{\nu}=\int_{0}^{\infty} d \omega \omega^{\nu} \frac{\operatorname{Im} \chi_{\sigma}(\omega)}{1-\mathrm{e}^{-\omega / T}},
$$

where the exponent ranges from $\nu=0$ for neutrino scattering to $\nu=7$ for the rate at which neutrinopair emission and absorption equilibrate the temperature of the nucleons with the temperature of the neutrinos and antineutrinos. For $\nu=0$ and provided the width $\left(1+G_{0}\right) / \tau_{\sigma}$ is small compared with $T$ (which is a necessary condition for the picture of long-lived quasiparticle excitations to be applicable, see also Ref. [3]), one can expand the denominator to first order in $\omega / T$ and the rate for neutrino scattering is then proportional to

$$
\int_{0}^{\infty} d \omega \frac{\operatorname{Im} \chi_{\sigma}(\omega)}{\omega} \sim \chi_{\sigma}(0)
$$

where the latter expression is a consequence of the Kramers-Kronig relation. Therefore, for this case the differences between the frequency dependences of $\chi_{\sigma}^{\text {relax }}$ and $\chi_{\sigma}$ have no effect. For larger values of $\nu$, higher frequencies are weighted more heavily than lower ones, and consequently the relaxation time approximation will overestimate rates. However, the error can never be greater than the maximum deviation of $\operatorname{Im} \chi_{\sigma}^{\text {relax }}$ from $\operatorname{Im} \chi_{\sigma}$.

There are a number of directions in which this work could be extended. One is to consider the case when $\omega \gtrsim T$, and another is to treat partially degenerate or nondegenerate nucleons.

\section{CONCLUDING REMARKS}

In this paper, we have solved the quasiparticle transport equation for the spin response of a normal Fermi liquid for arbitrary values of $\omega \tau_{\sigma}$. In a field-theoretical framework, this amounts to solving the Bethe-Salpeter 
equation for a quasiparticle-quasihole pair excitation, taking into account self-energy and vertex corrections at the level of two-body collisions. One of the advantages of the transport equation approach is that conservation laws are properly taken into account.

In particular, we have studied the relaxation of the isotropic part of the quasiparticle distribution function in momentum space. However, the basic formalism can be applied to relaxation of other spherical harmonic components, with appropriate changes in the definition of the quantity corresponding to $\lambda_{\sigma}$. These methods may also be applied to multicomponent systems, the main difference being that the coefficients $a_{r}$ then depend on the species, and one must solve a set of linear equations. The techniques therefore represent a generalization of those used to calculate transport coefficients of multicomponent Fermi liquids [25].

The differences between the exact solution and the relaxation time approximation are small compared with the uncertainty of other input to calculations of neutrino processes in dense matter. However, the effects could be of importance in related situations, such as the absorption of sound in liquid ${ }^{3} \mathrm{He}$ for conditions between the collisionless and hydrodynamic regimes. Another potential application is to calculate the frequency-dependent conductivity of a charged Fermi liquid and the magnetoresistance of metals, problems attacked in Ref. 7] by variational techniques.

\section{Acknowledgements}

We are grateful to Henrik Smith for helpful conversations and to John Avery for introducing us to the uses of Sturmian methods in other areas. We also thank Peter Orland and Jakob Yngvason for useful remarks. AS thanks the Niels Bohr International Academy and NORDITA for the warm hospitality. This work was supported in part by the Natural Sciences and Engineering Research Council of Canada (NSERC). TRIUMF receives funding via a contribution through the National Research Council Canada.

\section{APPENDIX A: MATHEMATICAL DETAILS}

To make the paper reasonably self-contained, we list a number of useful mathematical relations in this appendix. In the derivation of the quasiparticle transport equation, Eq. (18), we encountered the integrals

$$
K_{2}(x)=\int_{-\infty}^{\infty} \frac{d x_{2} d x_{4} \delta\left(x+x_{2}-x_{4}\right)}{4 \cosh \left(x_{2} / 2\right) \cosh \left(x_{4} / 2\right)},
$$

and

$$
K_{3}(x)=\int_{-\infty}^{\infty} \frac{d x_{2} d x_{3} d x_{4} \delta\left(x+x_{2}-x_{3}-x_{4}\right)}{8 \cosh \left(x_{2} / 2\right) \cosh \left(x_{3} / 2\right) \cosh \left(x_{4} / 2\right)} .
$$

These may be evaluated by representing the delta function as

$$
\delta(x)=\int_{-\infty}^{\infty} \frac{d t}{2 \pi} \mathrm{e}^{\mathrm{i} t x}
$$

and using the methods described in Appendix A of Chapter 1 of Ref. [11]. The results are

$$
K_{2}(x)=\frac{x}{2 \sinh (x / 2)},
$$

and

$$
K_{3}(x)=\frac{x^{2}+\pi^{2}}{2} \frac{1}{2 \cosh (x / 2)} .
$$

By multiplying Eq. (34) by $\widetilde{\Phi}_{r^{\prime}}(k)$, subtracting the same expression with $r$ and $r^{\prime}$ interchanged, and then integrating over $k$, one sees that the $\widetilde{\Phi}_{r}(k)$ are orthogonal in the sense that

$$
\int_{-\infty}^{\infty} d k \frac{\widetilde{\Phi}_{r}(k) \widetilde{\Phi}_{r^{\prime}}(k)}{\cosh ^{2}(\pi k)}=\delta_{r r^{\prime}} \frac{U_{r}}{2 \pi},
$$

where $U_{r}$ is given explicitly in Eq. (41). For the eigenfunctions in terms of $x$, this condition is equivalent to

$$
\int_{-\infty}^{\infty} d x\left(x^{2}+\pi^{2} \zeta^{2}\right) \Phi_{r}(x) \Phi_{r^{\prime}}(x)=\delta_{r r^{\prime}} \frac{U_{r} \Lambda_{r}}{2} .
$$

In the hydrodynamic limit $(\zeta \rightarrow 1)$, the eigenfunctions $\Phi_{r}(x)$ have a simple form, since

$$
\begin{aligned}
\Phi_{r}(x) & =\int_{-\infty}^{\infty} \frac{d k}{2 \pi} \widetilde{\Phi}_{r}(k) \mathrm{e}^{\mathrm{i} k x}, \\
& \sim \int_{-\infty}^{\infty} \frac{d k}{2 \pi} \operatorname{sech}(\pi k) C_{2 r}^{3 / 2}(\tanh (\pi k)) \mathrm{e}^{\mathrm{i} k x},
\end{aligned}
$$

where we have used Eq. (37) for $\widetilde{\Phi}_{r}(k)$. The integral can be evaluated by contour integration, closing the contour in the upper half plane for $x>0$ and in the lower half plane for $x<0$. The integrand has poles of order $2 r+1$ at $k=\mathrm{i}(\nu+1 / 2)$ with integer $\nu$, and therefore the integral is proportional to the sum of the residues of the integrand at these points, which is a geometrical progression with the ratio of subsequent terms equal to $-\mathrm{e}^{-|x|}$. The final result is $\Phi_{r}(x) \sim Q_{r}(x) /[2 \cosh (x / 2)]$, where $Q_{r}(x)$ is the residue of $\mathrm{e}^{\mathrm{i} k x} C_{2 r}^{3 / 2}(\operatorname{coth}(\pi k)) / \sinh (\pi k)$ at $k=0$, a polynomial of order $2 r$. The polynomials $Q_{r}(x)$ satisfy the orthogonality relation

$$
\int_{-\infty}^{\infty} d x \frac{x^{2}+\pi^{2}}{\cosh ^{2}(x / 2)} Q_{r}(x) Q_{r^{\prime}}(x)=0, \quad \text { for } \quad r \neq r^{\prime} .
$$

The first few eigenfunctions are given by

$$
\begin{aligned}
& Q_{0}(x) \sim 1 \\
& Q_{1}(x) \sim\left(1-\frac{5}{3} \frac{x^{2}}{\pi^{2}}\right), \quad \text { and } \\
& Q_{2}(x) \sim\left(1-\frac{14}{5} \frac{x^{2}}{\pi^{2}}+\frac{7}{15} \frac{x^{4}}{\pi^{4}}\right) .
\end{aligned}
$$


For other values of $\zeta$ the eigenfunctions do not have such a simple form.

[1] G. G. Raffelt, Stars as Laboratories for Fundamental Physics: The Astrophysics of Neutrinos, Axions, and Other Weakly Interacting Particles (University of Chicago Press, 1996).

[2] G. I. Lykasov, E. Olsson, and C. J. Pethick, Phys. Rev. C 72, 025805 (2005).

[3] G. I. Lykasov, C. J. Pethick, and A. Schwenk, Phys. Rev. C 78, 045803 (2008).

[4] L. D. Landau and I. Ya. Pomeranchuk, Dokl. Akad. Nuak. SSSR 92, 535 (1953); ibid. 92, 735 (1953); A. B. Migdal, Phys. Rev. 103, 1811 (1956).

[5] H. H. Jensen, H. Smith, and J. W. Wilkins, Phys. Lett. 27B, 532 (1968).

[6] J. Sykes and G. A. Brooker, Ann. Phys. (N.Y.) 56, 1 (1970); G. A. Brooker and J. Sykes, Ann. Phys. (N.Y.) 61, 387 (1970).

[7] L. E. G. Ah-Sam, H. H. Jensen, and H. Smith, J. Stat. Phys. 3, 17 (1971).

[8] E. Egilsson and C. J. Pethick, J. Low Temp. Phys. 29, 99 (1977).

[9] G. A. Brooker and J. Sykes, Ann. Phys. (N.Y.) 74, 67 (1972).

[10] J. Da̧browski and P. Haensel, Ann. Phys. (N.Y.) 97, 452 (1976).

[11] G. Baym and C. J. Pethick, Landau Fermi Liquid Theory: Concepts and Applications (Wiley, New York, 1991).

[12] We use units with $\hbar=k_{\mathrm{B}}=1$ throughout.

[13] D. Pines and P. Nozières, The Theory of Quantum
Liquids (Volume 1, Advanced Book Classics, Westview Press, 1999).

[14] A. A. Abrikosov and I. M. Khalatnikov, Zh. Eksp. Teor. Fiz. 32, 1083 (1957) [Sov. Phys. JETP 5, 887 (1957)]; Rep. Prog. Phys. 22, 329 (1959).

[15] Note that the average defined in Ref. [11, Eq. 1.2.88] is normalized to 2 .

[16] The quantity $\tau_{0}$ in this paper is denoted by $\tau$ in Ref. [11].

[17] L. D. Landau and I. M. Lifshitz, Quantum Mechanics (Pergamon, Oxford, 3rd Edition, 1977) p. 73.

[18] James Avery and John Avery, Generalized Sturmians and Atomic Spectra (World Scientific, Singapore, 2006).

[19] C. J. Pethick, Phys. Rev. 185, 384 (1969).

[20] Essentially the same result was obtained in studies of zero sound in liquid ${ }^{3} \mathrm{He}$ in Ref. [8, Eq. (60)].

[21] M. Abramowitz and I. A. Stegun, Handbook of Mathematical Functions (US Department of Commerce, 1970) p. 260.

[22] S. Bacca, K. Hally, C. J. Pethick, and A. Schwenk, arXiv:0812.0102

[23] A. Schwenk, B. Friman, and G. E. Brown, Nucl. Phys. A 713, 191 (2003).

[24] S. Hannestad and G. Raffelt, Astrophys. J. 507, 339 (1998).

[25] R. H. Anderson, C. J. Pethick, and K. F. Quader, Phys. Rev. B 35, 1620 (1987). 